\title{
Designing Fault-Tolerant Photovoltaic Systems
}

\author{
Xue Lin, Yanzhi Wang, and Massoud Pedram \\ University of Southern California \\ Jaemin Kim and Naehyuck Chang \\ Seoul National University
}

PV systems are subject to PV cell faults, which lower the output power and shorten the lifespan of the system. This article presents the design principles and runtime control algorithms of a fault-tolerant PV system which can detect and bypass PV cell faults in situ without any manual interventions.

Keywords: Photovoltaic System, fault-tolerance, reconfigurable PV panel

-Photovoltaic (PV) systems have received considerable attention thanks to the growing demand for renewable energy and the advancement of PV device technologies. PV systems have been widely deployed in electric vehicles, homes, power plants, and satellites.

The output power of a PV cell (also called solar cell) is dependent on the solar irradiance level and temperature. Figure 1 shows PV cell output current-voltage and power-voltage characteristics under different solar irradiance levels (Figure 1A) and temperatures (Figure 1B), where $G_{S T C}=1000 \mathrm{~W} / \mathrm{m}^{2}$ stands for the solar irradiance level under standard test condition [2]. On the PV cell output current-voltage curves, the solid black dots represent the maximum power points (MPP's) of a PV cell, which correspond to the peak power points on the PV cell output power-voltage curves. The maximum

This work was partly presented in the International Conference on Computer-Aided Design, 2012 [1].

This work is sponsored in part by the Software and Hardware Foundations program of the NSF's Directorate for Computer \& Information Science \& Engineering, the Center for Integrated Smart Sensors funded by the Ministry of Science, ICT \& Future Planning as Global Frontier Project (CISS-2012054193). The SPORT lab at University of Southern California and the ICT at Seoul National University provide the research facilities for this study.

Direct questions and comments about this article to Massoud Pedram, University of Southern California, Los Angeles, CA 90089, USA (email: pedram@usc.edu) and Naehyuck Chang, Seoul National University, Seoul 151-742 Korea (email: naehyuck@elpl.snu.ac.kr). output power of a PV cell increases as solar irradiance increases and temperature decreases.
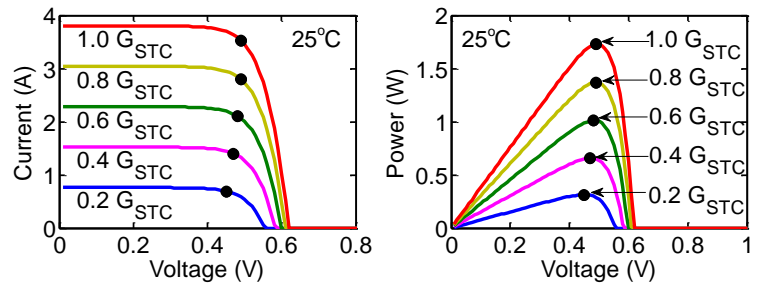

(A) PV cell output under different solar irradiance.
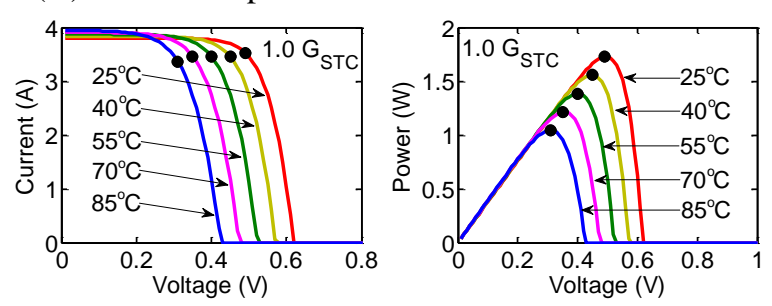

(B) PV cell output under different temperature.

Figure 1. PV cell output characteristics.

Figure 2 shows a typical PV system architecture, which comprises a PV panel, a charger, and a load device (a battery used to store harvested energy). The PV panel consists of $N \times M(=4 \times 4)$ identical PV cells, where $M \mathrm{PV}$ cells are connected in parallel to form a $P V$ cell group and $N$ PV cell groups are connected in series to form the whole panel. If all of the PV cells in the panel simultaneously operate at their MPP's, the PV panel achieves the maximum output power. The maximum power point tracking (MPPT) technique can adaptively 
adjust the output current of the PV panel to ensure that the panel produces the maximum output power under varying solar irradiance and temperature conditions [3]. The charger is implemented using a DC-DC switching converter [4]. The input ports of the charger are connected to the PV panel, whereas its output ports are connected to the battery. The power consumption of the charger is reduced when the input and the output voltages of the charger are close, and the charger output current is within some range.

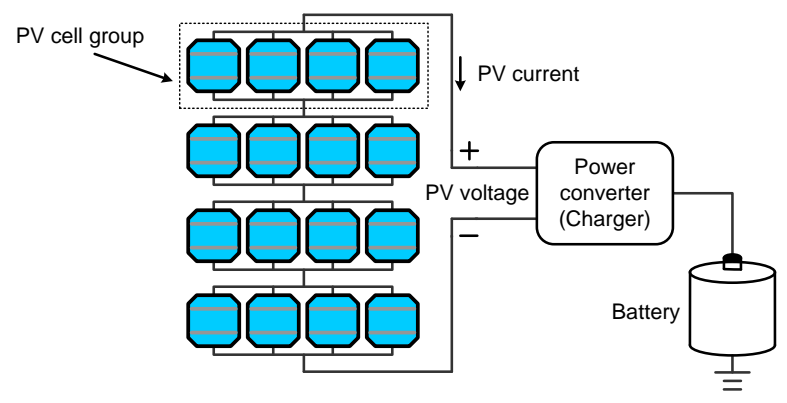

Figure 2. A typical PV system architecture.

口PV cell faults in a PV system are caused by contact failure, corrosion of wire, hail impact, moisture, etc [5]. Authors in [6] conducted a two-year monitoring study of $27 \mathrm{PV}$ systems and recorded the annual occurrence rates of PV cell faults in the range of $1.1 \% \sim 11.7 \%$. Due to the increasing number of orbital debris, the fault occurrence rate on PV systems in space is even higher [7]. A PV cell fault is equivalent to an open circuit at the PV cell position. A PV cell fault leads to a reverse bias on the other PV cells in the same PV cell group. This results in creation of hot spots in the PV panel, which can give rise to even more PV cell faults. Integration of bypass diodes with PV cells can solve the reverse bias problem [8], a technique that we also employ.

However, bypass diodes do not address the severe output power degradation of the PV system due to PV cell faults. In particular, the output power loss due to faulty PV cells is much higher than what one expects by a simple counting of healthy and faulty cell numbers. For example, for the $4 \times 4 \mathrm{PV}$ panel in Figure 2 , with one PV cell fault, simple counting argument would indicate an output power degradation of $1 / 16=6.25 \%$. In reality, the output power degradation due to one PV cell fault is $16.5 \%$, because PV cells in a PV panel cannot continue to work at their MPP's when the balanced structure of a PV panel is broken by the faults.

Unfortunately, manual fault detection and elimination are expensive and almost impossible for remote PV systems (e.g., PV systems in orbital or deep space missions.) Therefore it is necessary to design a fault-tolerant PV system in the sense that an embedded system controller can dynamically detect and bypass PV cell faults.

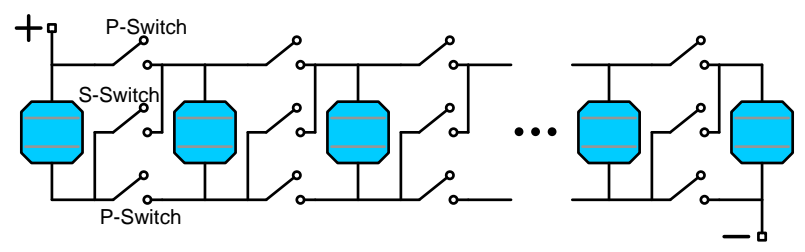

(A)
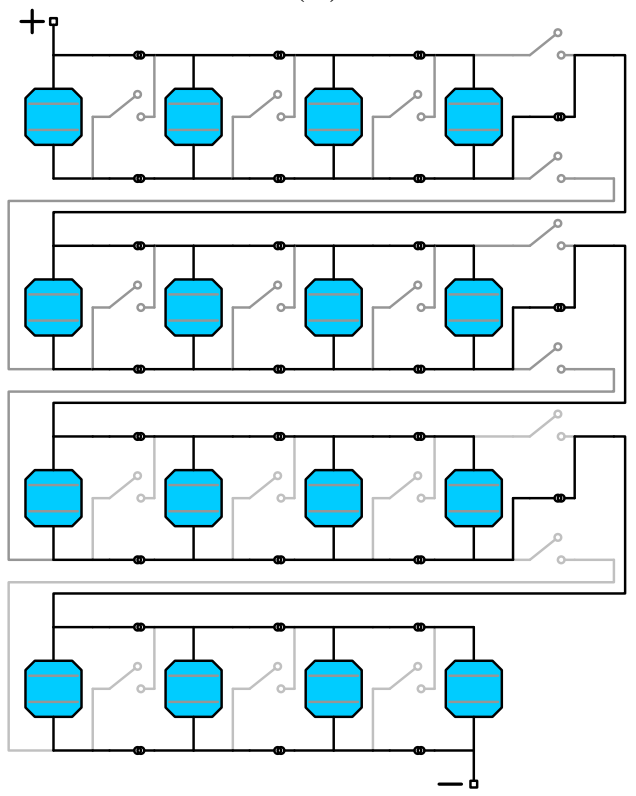

(B)

Figure 3. (A) The reconfigurable $P V$ panel structure, and (B) a $4 \times 4$ configuration of a PV panel.

- Fault-tolerant PV systems are desirable for longer service life of PV systems. Several PV cell fault diagnosis techniques have been proposed [9], [10], [11]. While these techniques can detect the PV cell faults, they need additional equipment (e.g., signal generators) for fault diagnosis, cannot accurately locate faults on the panel, or lack an effective fault bypassing mechanism. This is not surprising because these fault diagnosis 
techniques are generally bound by the fixed (non-programmable) structure of the PV panel.

\section{Reconfigurable PV Panel}

This article presents the design of a fault-tolerant PV system, utilizing a reconfigurable PV panel structure as depicted in Figure 3A. We introduced this PV panel structure for combating the partial shading effect by realizing imbalanced PV cell connection topologies within the PV panel [12]. We propose to use this same reconfigurable PV panel structure for the fault detection and fault bypassing in this article.

In the proposed reconfigurable PV panel structure, each PV cell except for the last one is integrated with two $P$-switches and one $S$-switch. Figure 3A shows the electrical connectivity of the PV cells and switches (rather than their actual locations within the panel). By controlling the ON/OFF states of the switches, we can achieve the $N \times M \mathrm{PV}$ panel configuration for normal system operation. Figure $3 B$ illustrates how to achieve the $4 \times 4$ configuration in Figure 2.

The PV panel for normal system operation is optimized such that the output voltage of the panel at its MPP matches the voltage of the battery. In this way, the charger consumes the least power and the output power of the PV system is maximized. However, the MPP voltage of a PV cell may change due to aging, partial shading, and temperature variation. In these cases, we can reconfigure the PV panel to increase the PV system output power. For example, we can change from a $4 \times 4$ configuration into a $8 \times 2$ one. This reconfigurability enables more efficient PV system operation under various environmental conditions. More importantly, the reconfigurable PV panel enables us to perform fault detection and bypassing as detailed next.

\section{Reconfiguration for Fault Detection and Fault Bypassing}

The objective of fault detection is to identify any PV cell faults in the PV panel. Fault bypassing aims at forming a new PV panel configuration to minimize the output power loss caused by PV cell faults. Consider a PV panel assuming a $N \times M$ configuration during normal system operation. For fault detection, we may need to form a $k \times M \mathrm{PV}$ panel configuration $(k \leq N)$ with a selected set of PV cells and measure their combined output power to determine whether a PV cell fault exists in this portion of PV panel. During fault bypassing, we may need to form a $N_{o p t} \times M_{o p t}$ PV panel configuration to improve the system output power, where the faulty PV cells, and perhaps some healthy PV cells are excluded from the new configuration $\left(N_{o p t} \times M_{o p t}<N \times M\right)$.

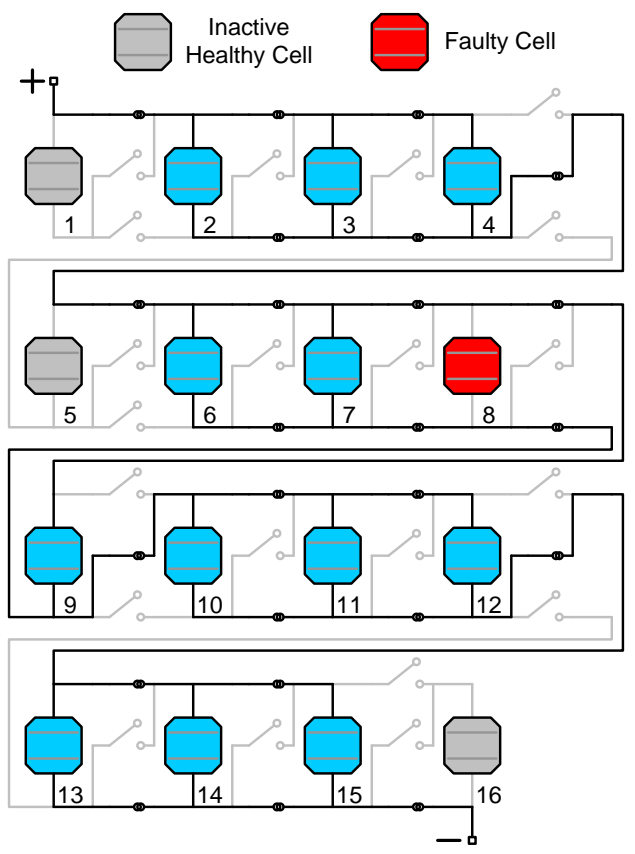

Figure 4. Example of PV panel reconfiguration during fault detection and bypassing.

Inactivating even healthy PV cells may become necessary in some cases of fault bypassing. For example, suppose we have $42 \mathrm{PV}$ cells and one of them is faulty. We cannot form a good configuration with a prime number of 41 healthy PV cells such that the MPP voltage of this configuration matches with the battery voltage. Therefore, we would like to inactivate one healthy PV cell to have 40 active cells. Then we are able to perform a $5 \times 8$ or $8 \times 5$ configuration to increase the PV system output power. The inactivated healthy PV cell may be used later if we encounter an additional PV cell fault.

We use Figure 4 as an example to illustrate how to effectively control the ON/OFF states of the switches for fault detection and fault bypassing. PV cells 1, 5, 16 are inactive healthy PV cells, and PV cell 8 is a faulty PV 
cell. Figure 4 shows a $4 \times 3 \mathrm{PV}$ panel configuration formed with the remaining PV cells. The faulty PV cell 8 is open-circuited and inactive outside our control. The healthy PV cells can be isolated in either of two cases: (i) they are located between two PV cell groups; (ii) they are at the leftmost or the rightmost position in the electrical connection of the PV panel (Figure 3A). In Figure 4, an example of the first case is PV cell 5, whereas examples of the second case are PV cells 1 and 16.

\section{Fault Existence Checking:}

1. Form a $k \times M$ configuration with the designated $k \times M$ PV cells.

2. Calculate the theoretical output power $P_{\text {the }}$ of this $k \times M$ configuration assuming no $\mathrm{PV}$ cell fault among them.

3. Track the actual output power $P_{a c t}$ of this PV panel configuration using the MPPT technique.

4. If $P_{a c t}<P_{\text {the }}-\varepsilon$, where $\varepsilon$ denotes a pre-specified error threshold, then there exists a PV cell fault among these PV cells.

Return 1.

Else

Return 0.

-The fault detection and bypassing algorithms for fault-tolerant PV systems are proposed based on the reconfigurable PV panel structure. The fault detection algorithm can identify a PV cell fault with logarithmic time complexity or determine the non-existence of a PV cell fault in O(1) time. The fault bypassing algorithm determines the optimal configuration of a PV panel, such that the PV system output power degradation due to PV cell faults can be minimized.

The fault detection algorithm is executed every $\Delta t$ units of time. $\Delta t$ must be much smaller than the average fault occurrence time interval, which is in the order of days or months [6], so that we can safely assume that at most one PV cell fault occurs during each time interval $\Delta t$ and the fault detection algorithm only needs to detect at most one newly-occurring fault at each execution. The fault detection algorithm first compares the actual PV panel output power with the theoretical output power of the PV panel without any faults. If the difference is smaller than a pre-specified error threshold, then there will exist no new fault, and the fault detection algorithm will terminate in $\mathrm{O}(1)$ time. Otherwise, the fault detec- tion algorithm will continue to find the fault, and the fault bypassing algorithm will be executed. In most cases, the fault detection algorithm will confirm the non-existence of a new fault. Therefore, the computational overhead of the fault detection and bypassing algorithms is small. In practice, we have found that $\Delta t$ can be set to an hour.

\section{Fault Detection Algorithm}

The basic step of the fault detection algorithm is the Fault Existence Checking algorithm, which determines whether a PV cell fault exists in a set of $k \times M$ PV cells. We track the maximum output power of the $k \times M \mathrm{PV}$ panel configuration using the charger in the PV system at Step 3 of the Fault Existence Checking algorithm. In reality, $k$ must be larger than or equal to a threshold value $K_{\min }$ such that the output voltage of the $k \times M \mathrm{PV}$ panel configuration is high enough to properly drive the charger. This means that the Fault Existence Checking algorithm cannot run on a PV panel configuration smaller than $K_{\min } \times M$.

The fault detection algorithm has two steps: first, determine which row the faulty PV cell is located at (row search); second, determine which column the faulty PV cell is located at (column search). To find the location of the potential faulty PV cell in the $N \times M$ PV panel, we first run the Fault Existence Checking algorithm on the whole PV panel. If it is confirmed that no PV cell fault exists, the fault detection algorithm will terminate. Otherwise, the fault detection algorithm will continue to find the location of the PV cell fault as explained next.

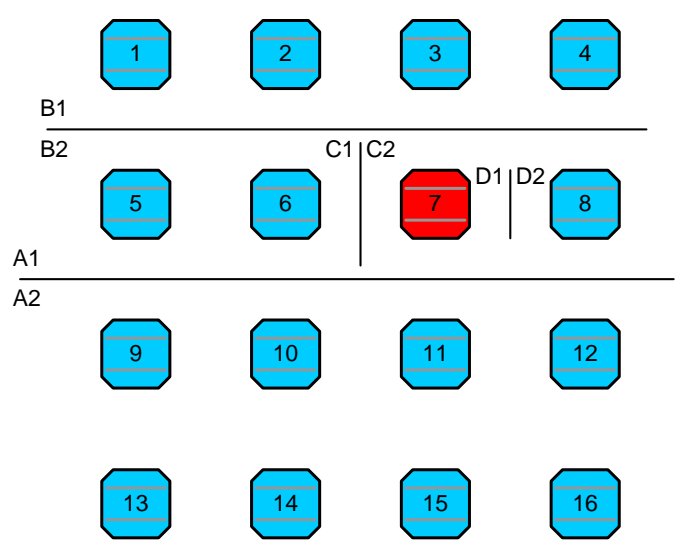

Figure 5. Demonstration of the fault detection algorithm. 
We use Figure 5 to demonstrate how the row search and column search proceed. In this example, $N=4$, $M=4$, and $K_{\min }=2$. For row search, we bisect the PV panel into the first two rows (A1) and the remaining two rows (A2). We run the Fault Existence Checking algorithm on A1 and find out that A1 contains a faulty PV cell. Then we bisect A1 into the first row (B1) and the second row (B2). The size of B1 is smaller than $K_{\text {min }} \times M$. Therefore, we form a $K_{\min } \times M(2 \times 4)$ configuration from B1 along with the third row, which has been confirmed to contain only healthy PV cells, and subsequently, run the Fault Existence Checking algorithm on this configuration. We determine that B1 does not contain the faulty PV cell, and therefore, the faulty PV cell is within B2. Now we have located the row containing the faulty PV cell.

For column search, we bisect B2 into PV cells 5 and 6 (C1) and PV cells 7 and 8 (C2). We run the Fault Existence Checking algorithm on $\mathrm{C} 1$ along with $\mathrm{PV}$ cells 3 , 4, 9 12 that are confirmed healthy. We pick these healthy PV cells, because in this way we can form a $2 \times 4$ configuration, with PV cells 7 and 8 bypassed between the first PV cell group (PV cells 3 6) and the second PV cell group (PV cells 9 12). We find out that $\mathrm{C} 1$ does not contain the faulty PV cell, and therefore, $\mathrm{C} 2$ contains the faulty PV cell. We bisect $\mathrm{C} 2$ into PV cell 7 (D1) and PV cell 8 (D2). We form a $2 \times 4$ configuration from D1 along with PV cells 4 6 and 9 12, and run the Fault Existence Checking algorithm on this configuration. We confirm that the faulty PV cell is PV cell 7, and thereby, conclude the column search.

\section{Fault Bypassing Algorithm}

The fault bypassing algorithm determines the optimal configuration of a PV panel, such that the PV system output power loss due to PV cell faults is minimized. We need to decide (i) the number of active healthy PV cells $S$, and (ii) the optimal $\mathrm{PV}$ panel configuration $N_{\text {opt }} \times$ $M_{\text {opt }}(=S)$. Let us denote the number of factors of $S$ by $F(S)$. The maximum output power of a PV panel is approximately proportional to $S$. And a $S$ value is preferred if $F(S)$ is larger, since we have more choices of PV panel configurations with this $S$ value.

Assume that the PV panel has $N \times M$ PV cells and $L$ PV cell faults have been identified so far. Therefore, we have $S_{\max }=N \times M-L$. First, we determine a set of candidate $S$ values in ascending order, which satisfies $S+F(S) \geq S_{\max }+F\left(S_{\max }\right)$. There are $F(S)$ possible configurations using $S$ active healthy PV cells. Among these configurations, there exists an optimal configuration that provides maximum PV system output power $P_{\text {max }}(S)$. Based on the PV cell and charger model, we find the optimal $S_{\text {opt }}$ value by ternary search on the set of candidate $S$ values, such that $P_{\max }\left(S_{\text {opt }}\right)$ is the maximum achievable PV system output power. The optimal PV panel configuration is determined accordingly.

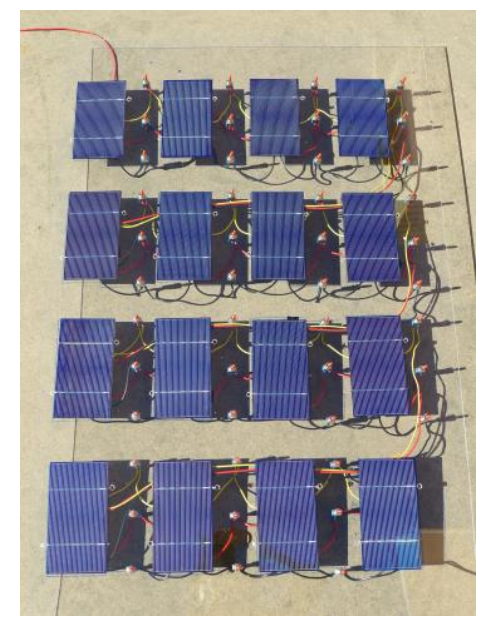

Figure 6. Prototype of the fault-tolerant PV system.

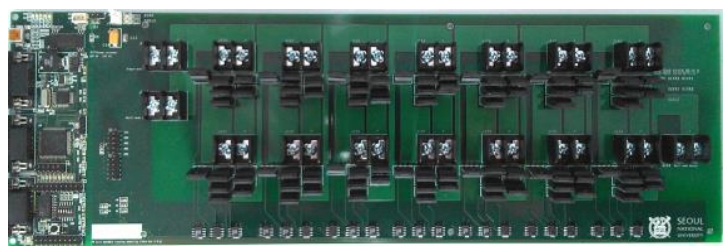

(A)

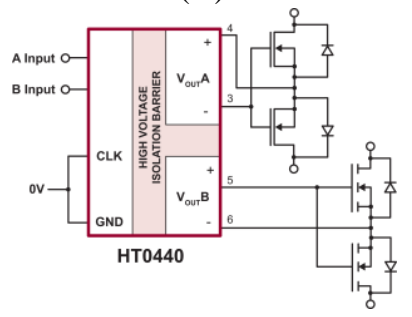

(B)

Figure 7. (A) Computer-controlled programmable switch board, and (B) semiconductor realization of the switches. 
A prototype of the fault-tolerant PV system is implemented to substantiate the feasibility and effectiveness of our structure design and control algorithms. Figure 6 shows the prototype of the reconfigurable PV panel. The PV panel consists of 16 PV cells, each of which (except for the last PV cell) is integrated with three toggle switches. PV cells and toggle switches are mounted on top of an acrylic board, whereas connection wires are routed in the back of the board. A PV cell fault can be emulated by blocking the surface of a PV cell, since zero solar irradiance on a PV cell results in zero output current, which is equivalent to a PV cell fault.

Toggle switches in the prototype are operated manually to demonstrate the idea of fault-tolerant PV systems. In addition, we have the design of computer-controlled programmable switch set (Figure 7A), which can be integrated into large-scale PV systems. The switches are realized by a back-to-back connection of power MOSFETs (Figure 7B).

We perform the fault detection algorithm on this PV panel based on Fault Existence Checking algorithm. We set the fourth PV cell in the first row as a PV cell fault by blocking the surface of it. First, when we measure the maximum output power of the PV panel, we observe an $18 \%$ output power degradation on the prototype, which is quite near to the $16.5 \%$ power degradation from simulation. Secondly, when we measure the maximum output power of the first two rows of the PV panel, we observe a $23 \%$ output power degradation, which is again quite close to the $19.7 \%$ power degradation from simulation. We proceed until finding the location of the PV cell fault. Details are omitted due to space limitation. It demonstrates that Fault Existence Checking algorithm effectively detects the existence of a PV cell fault.

We further perform the fault bypassing algorithm on the panel. We reconfigure the PV panel into a $3 \times 5$ configuration with the PV cell fault bypassed. We observe a $10 \%$ output power improvement over the original $4 \times 4$ configuration with one PV cell fault, while the theoretical output power improvement should be $12 \%$. In another testing example, we block the four PV cells in the bottom right corner of the panel to emulate PV cell faults. Then we reconfigure the PV panel into a $4 \times 3$ configuration with the PV cell faults bypassed. We observe a $35 \%$ output power improvement over the original $4 \times 4$ configuration with four PV cell faults, while the theoretical output power improvement is $37 \%$.
- Large-scale PV system simulation is performed to compare the performance of the fault-tolerant PV system to that of the baseline PV system without any fault-tolerant design. We extract the PV cell model from the prototype. The fault-tolerant PV system employs the reconfigurable PV panel structure with $100 \mathrm{PV}$ cells, and the baseline PV system has the fixed $20 \times 5$ PV panel.

Figure 8 shows the output power of the fault-tolerant PV system and the baseline system as a function of the number of PV cell faults. For a given number of PV cell faults, 100 groups of the locations of PV cell faults are randomly generated to simulate the occurrences of PV cell faults. The red dots represent the output power of the fault-tolerant PV system, which is only related to the number of PV cell faults. The blue dots present the output power of the baseline system, which is related to both the number of PV cell faults and the locations of PV cell faults. The fault-tolerant PV system always outperforms the baseline system. Depending on the locations of PV cell faults, the output power of the baseline system may degrade significantly. If all the PV cell faults happen to the same PV cell group, the output power degradation of the baseline system is the most significant. In the fault-tolerant PV system, the output power degradation due to PV cell faults is kept small.

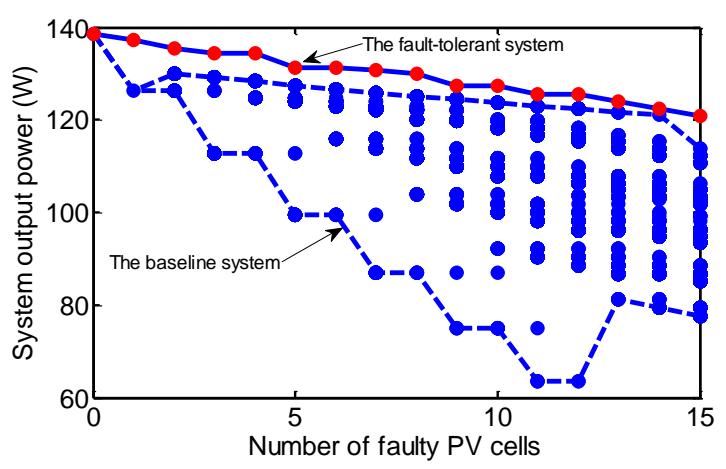

Figure 8. Comparison between the fault-tolerant PV system with the baseline system.

- In this article we proposed the design of fault-tolerant PV systems. The proposed fault-tolerant PV systems can be applied for remote PV systems, e.g., PV systems in space, where manual PV cell fault detection and elimination are expensive and almost impossi- 
ble. The fault-tolerant PV systems can minimize side effects of PV cell faults to increase the robustness.

\section{QReferences}

[1] X. Lin, et al., "Online fault detection and tolerance for photovoltaic energy harvesting systems," in Proc. ICCAD, 2012.

[2] Y. Wang, et al., "Enhancing efficiency and robustness of a photovoltaic power system under partial shading," in Proc. ISQED, 2012.

[3] N. Femia, et al., "Optimization of perturb and observe maximum power point tracking method," IEEE Trans. Power Electronics, 2005.

[4] Y. Wang, et al., "Charge migration efficiency optimization in hybrid electrical energy storage (HEES) systems," in Proc. ISLPED, 2011.

[5] A.M. Bazzi, et al., "Fault impacts on solar power unit reliability," in Proc. APEC, 2011.

[6] S.K. Firth, et al., "A simple model of PV system performance and its use in fault detection," Solar Energy, 2010.

[7] V.M. Smirnov, et al., "Study of micrometeoroid and orbital debris effects on the solar panels retrieved from the space station MIR," Space Debris, 2000.

[8] M.S. Swaleh, et al., "Effect of shunt resistance and bypass diodes on the shadow tolerance of solar cell modules," Solar Cells, 1982.

[9] A. Houssein, et al., "Monitoring and fault diagnosis of photovoltaic panels," in Proc. EnergyCon, 2010.

[10] K.H. Chao, et al., "Modeling and fault simulation of photovoltaic generation systems using circuit-based model," in Proc. ICSET, 2008.

[11] L. Schirone, et al., "Fault finding in a $1 \mathrm{MW}$ photovoltaic plant by reflectometry," in Proc. WCPEC, 1994.

[12] X. Lin, et al., "Near-optimal, dynamic module reconfiguration in a photovoltaic system to combat partial shading effects," in Proc. DAC, 2012.

Xue Lin is a PhD student in the Department of Electrical Engineering at University of Southern California. She got the B.E. degree from Tsinghua University in 2009. Her research interests include photovoltaic (PV) system management, near-threshold computing, and low power system design.

(3740 McClintock Ave. EEB 338, Los Angeles, CA, 90089. Email: xuelin@usc.edu. Phone: 213-740-4441.)

Yanzhi Wang is a $\mathrm{PhD}$ student in the Department of EE, University of Southern California. He got the B.E. degree in Department of Electronic Engineering, Tsinghua
University, 2009. He has published over 50 papers in low power system design, energy generation and storage, etc. (3740 McClintock Ave. EEB 338, Los Angeles, CA, 90089, USA. Email: yanzhiwa@usc.edu. Phone: 213-740-4441.)

Massoud Pedram is the Stephen and Etta Varra Professor of EE at USC. He received his B.S. degree in EE from Caltech in 1986 and $\mathrm{PhD}$ in EECS from UC-Berkeley in 1991. He is an IEEE Fellow, an ACM Distinguished Scientist, and Editor-in-Chief of the ACM TODAES and IEEE JETCAS.

(3740 McClintock Ave. EEB 344, Los Angeles, CA, 90089, USA. Email: pedram@usc.edu. Phone: 213-740-4458.)

Jaemin Kim is a $\mathrm{PhD}$ student in Dept. of Computer Science and Engineering at Seoul National University. His research interests include low-power embedded system design and photovoltaic system. He received a B.S. degree and an M.S. degree in computer science and engineering from Seoul National University, Seoul, Korea.

(Building 302, Room 431, School of Computer Science and Engineering, College of Engineering, Seoul National University, 599 Kwanak-Ku-Kwanak Road, Seoul, 151010, Korea. Email: jmkim@elpl.snu.ac.kr. Phone: 82-(2)-880-1834.)

Naehyuck Chang is a professor in Dept. of Computer Science and Engineering at Seoul National University. His research interest includes low-power systems design and energy systems. He is the ACM SIGDA Chair, an ACM Distinguished Scientist, and an IEEE Fellow.

(Building 302, Room 431, School of Computer Science and Engineering, College of Engineering, Seoul National University, 599 Kwanak-Ku-Kwanak Road, Seoul, 151010, Korea. Email: naehyuck@elpl.snu.ac.kr. Phone: 82-(2)-880-1834.) 